\title{
Updates on drug discovery in ovarian cancer
}

\author{
Steven J Gibson ${ }^{1,2}$, Krishnansu S Tewari ${ }^{3}$, Bradley J Monk $k^{1,2}$ and Dana M Chase ${ }^{1,2^{*}}$
}

\begin{abstract}
Drug discovery in the ovarian cancer arena continues to launch important new clinical trials. Many biologic agents are being studied in phase II and phase III clinical trials for recurrent disease. These agents include compounds that disrupt angiogenesis through a variety of mechanisms. Other oncogenic pathways are also specifically targeted such as PARP, MEK, and topoisomerase inhibitors which are currently being studied in phase III trials. Various cytotoxic agents, as well as therapeutic vaccines, are also under investigation, and continue to demonstrate promising new data. The relevant agents in the treatment of ovarian cancer which have demonstrated positive phase II activity will be discussed.
\end{abstract}

Keywords: Ovarian cancer, Chemotherapy, Targeted therapy, Angiogenesis, Recurrent cancer, Clinical trials

\section{Review}

Remissions after primary therapy in ovarian cancer are usually short-lived. Although intially responsive to a platinum and taxane-based therapy, recurrent disease is difficult to treat. Furthermore, there are few approved agents to treat recurrent ovarian cancer. Although patients that recur after 6 to 12 months of initial treatment may be retreated with a platinum plus taxane, those who relapse earlier or develop significant toxicity, may be given pegalated lipsomal doxorubicin, gemcitabine (in combination with platinum), etoposide, alkeran, topotecan, and/or hexamethylmelamide [1]. Unfortunately the response rate to these agents is generally less than $30 \%$, and demonstrable survival benefits have not been shown. With the introduction of targeted drugs, such as trastuzumab in breast cancer, strategies in drug development have focused on the development of biologic agents that demonstrate selectivity for tumor tissue.

\section{Introduction}

In 2010, we published on recent advances in drug discovery for ovarian cancer [1]. Since then, multiple drugs have either failed to advance into further development, have newly been developed, or have demonstrated activity in phase III trials. For example, with respect to

\footnotetext{
* Correspondence: Dana.chase@chw.edu

${ }^{1}$ The Division of Gynecologic Oncology, University of Arizona Cancer Center, 500 West Thomas Road, Suite 600, Phoenix, AZ 85013, USA

${ }^{2}$ Creighton University School of Medicine, St. Joseph's Hospital and Medical

Center, A Dignity Health Member, 500 West Thomas Road, Suite 600,

Phoenix, AZ 85013, USA

Full list of author information is available at the end of the article
}

bevacizumab, several positive phase III trials have supported the use of this drug in upfront and recurrent ovarian cancer cases yet FDA approval is pending. Another example includes a 940 patient, phase III AGOOVAR16 study which proved advantageous in ovarian cancer treatment with pazopanib, increasing median progression-free survival (PFS) by about 5.6 months [2]. In addition, trabectedin was previously discussed and positive phase III activity was reported, improving PFS, and overall response rate in a 672 patient study [3]. Lastly, phase III results from the TRINOVA- 1 trial of over 900 patients found that trebananib (AMG 386) increased PFS as well as reduced disease progression and death by $34 \%$ when combined with paclitaxel. Unfortunately, several of the drugs previously described have been found to be inactive, or with disappointing clinical outcomes. This review will thus highlight new drugs for ovarian cancer that have recently demonstrated positive phase II activity (Table 1). The ultimate goal with this type of drug development is to achieve prolonged remission and improved quality of life (QOL), for patients with recurrent ovarian cancer.

\section{Targeted agents \\ Angiogenesis inhibitors \\ VEGF-dependent}

Vascular endothelial growth factor (VEGF) is a signaling molecule involved in triggering the growth of blood vessels within cancers. The VEGF mechanism of action encompasses binding to tyrosine kinase transmembrane 
Table 1 Updates in ovarian cancer drug discovery demonstrating positive phase II activity

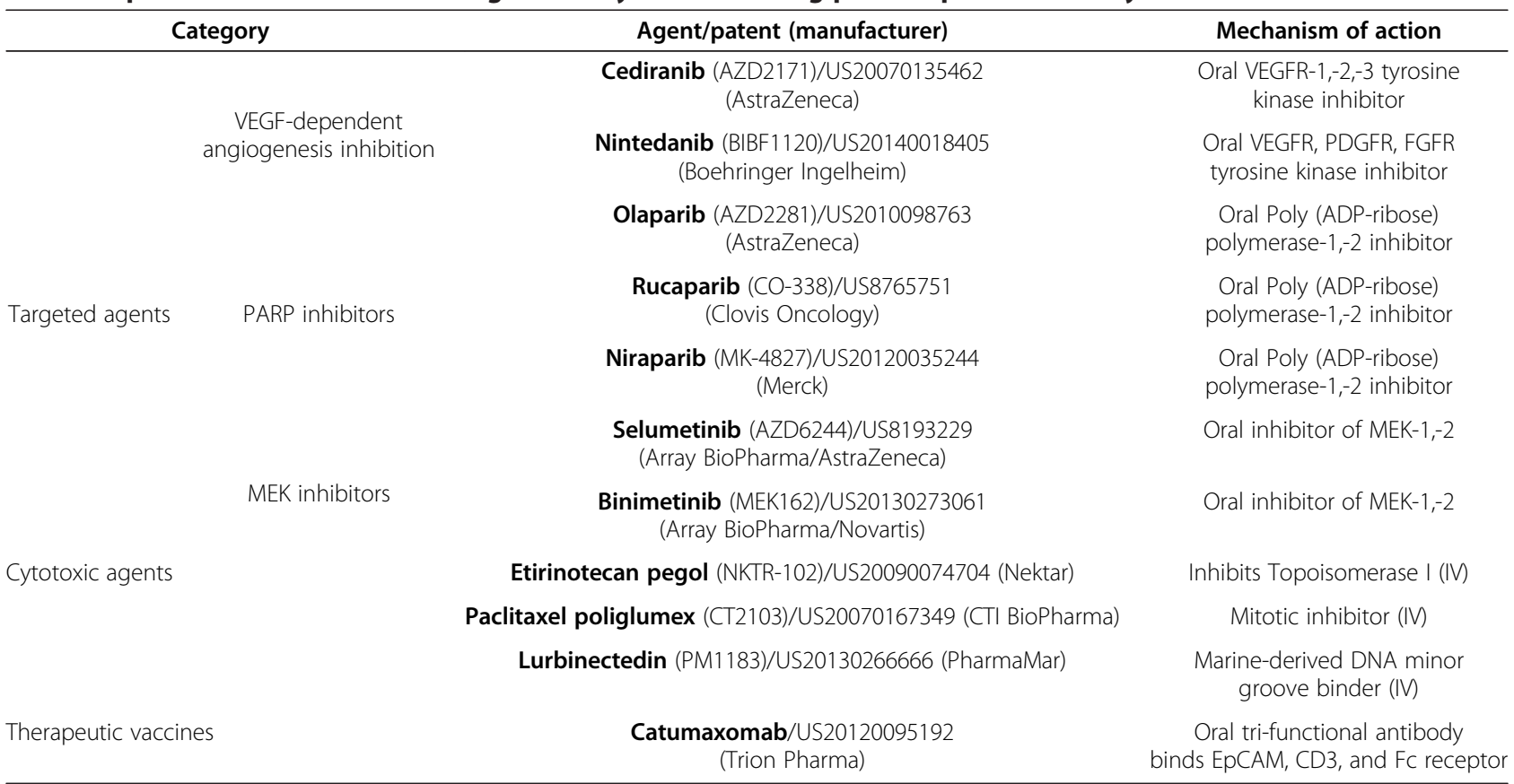

receptors (VEGFR), found on tumor endothelial cells, initiating angiogenesis (Figure 1) [4]. VEGFR-2 regulates cellular VEGF interactions, making it a crucial component in the angiogenic process. Modulating VEGF has become a highlighted area of study with potential in therapeutic interventions.

\section{VEGFR-1,-2,-3 inhibitor: cediranib (AZD2171)}

While multiple phase III trials of cediranib (Recentin ${ }^{\mathrm{TM}}$ ) may have been disappointing for colon cancer drug discovery, the drug has gained noticeable attention in the treatment of ovarian cancer [5,6]. Cediranib is a potent anti-angiogenesis agent that acts by blocking the VEGF signaling cascade via the inhibition of all three VEGFR tyrosine kinases, thus preventing the formation of tumor vasculature [7].

Cediranib was evaluated in combination with the PARP inhibitor, olaparib, in the first ovarian cancer trial to utilize two orally-administered investigational drugs [8]. The multi-center, phase II study comparing olaparib

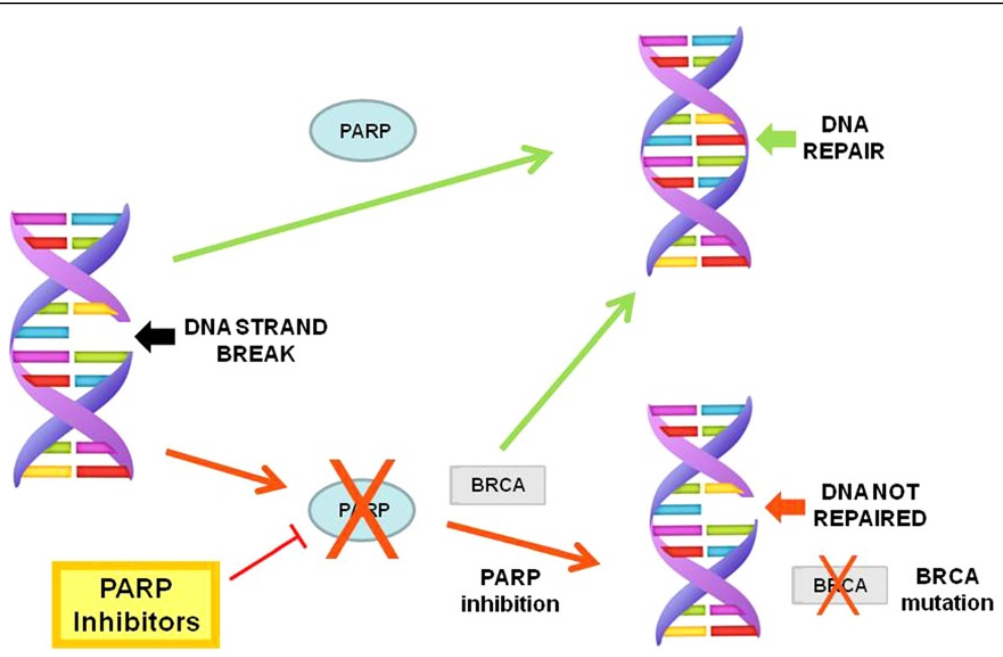

Figure 1 Poly(ADP-Ribose) polymerase (PARP) inhibitors. 
(400 mg twice daily) to cediranib (30 mg daily) plus olaparib (200 mg twice daily) found that the combination had nearly doubled PFS (9 vs 17.7 months). Of the 90 platinum-sensitive or BRCA-mutation patients in the study, significantly more had an objective response rate (ORR) in the combination group (56\% vs $84 \%)$. With these improvements in PFS and ORR came an increase in grade $3 / 4$ toxicities, with about ten times as many observed with the combination (7\% vs 70\%) [9]. The ICON6 phase III European trial found similar improvements in PFS and overall survival (OS) when cediranib was combined with platinum-based chemotherapy [10]. With these encouraging results, the planning of a phase III cediranib and olaparib combination trial is underway [8].

\section{VEGF receptor, platelet-derived and fibroblast growth factor receptor: Nintedanib (BIBF 1120)}

The additional targeting of proangiogenic receptors continues to be of interest, and has been proposed to improve the efficacy of VEGF blockade. Nintedanib is a triple angiokinase inhibitor that simultaneously blocks the VEGF, platelet-derived, and fibroblast growth factor receptors [11]. When studied in animal tumor models, nintedanib effectively reduced tumor blood vessel density and integrity [12].

A randomized, placebo-controlled phase II trial evaluated nintedanib maintenance therapy (250 mg for 36 weeks), after chemotherapy in patients with relapsed ovarian cancer. Eighty-three women were enrolled and following the treatment cycle, the PFS was $16.3 \%$ for nintedanib patients and $5 \%$ for placebo patients. Nintedanib patients experienced a much higher rate of grade 3 or 4 hepatotoxicity (51.2\%), compared to that of the placebo group (7.5\%) [13]. The potential effect of nintedanib nearly tripling PFS, when compared to the placebo, has warranted a 1,300 patient, phase III study of this drug in the LUME-Ovar 1 trial [14]. Phase III trials of nintedanib are also currently underway for non-small cell lung cancer and being planned for hepatocellular, renal, and colorectal cancers [12].

\section{Poly (ADP-ribose) polymerase inhibitor Olaparib (AZD2281)}

Poly(ADP-ribose) polymerases (PARPs) are proteins involved in the repair of DNA [15]. PARPs assist in the repair of DNA single-strand breaks by repairing base excisions. BRCA1 and BRCA2 proteins involved in DNA recombination play crucial roles in repairing doublestrand breaks, and can do so in the setting of PARP inhibition. However, PARP inhibition in BRCA-deficient cells results in the incapability to repair DNA damage induced by chemotherapy. In a BRCA-deficient environment, cell death can manifest when DNA breakage is not repaired, and the cell is exposed to such agents as PARP inhibitors that hinder single-strand break repair (Figure 1) [16].
BRCA mutations represent a minority of breast and ovarian cancers. These homozygous mutations, that are unique to the tumor cells, result in the inability to repair DNA which then can be exclusively targeted by a PARP inhibitor, preserving the patient's non-tumor cells. Preclinical studies discussed by Fong et al. [17] demonstrate that BRCAdeficient cells were 1000-fold more sensitive to PARP inhibitors. Ledermann et al. supported these findings in subgroup analysis of a 265 patient study evaluating olaparib (400 mg) maintenance therapy in platinum-sensitive, relapsed ovarian cancer. The original phase II study found PFS to be significantly longer with olaparib maintanence than placebo ( 8.4 vs. 4.8 months, respectively) [18]. A preplanned, subgroup analysis showed patients with a BRCA mutation (BRCAm) had the greatest clinical benefit, specifically germline BRCAm which had a 7.1 month increase in PFS and significant improvement in QOL. They found an average 6.9 month increase in PFS, an 8.5 month improvement in time to second disease progression, and a three month increase in OS in BRCAm patients when compared with placebo [19].

These results have initiated two phase III (SOLO) trials [20]. The U.S.-based SOLO1 trial, will examine olaparib (300 mg twice daily) as maintenance therapy in 2,500 BRCAm ovarian cancer patients following firstline platinum chemotherapy [21]. The European-based SOLO2 trail will examine the same dosage of olaparib maintenance therapy but instead in 440 BRCAm patients with platinum-sensitive, relapsed, or recurrent ovarian cancer [22].

A separate, randomized, phase II study also found significant improvement in PFS with the addition of olaparib to paclitaxel and carboplatin followed by subsequent maintenance therapy (12.2 vs. 9.6 months placebo). The ORR remained fairly similar (64\% vs. 58\%), and the most common adverse events reported in the combination phase were alopecia, nausea, and fatigue [23].

\section{Rucaparib (CO-338)}

Rucaparib is an inhibitor of poly(ADP-ribose) polymerases 1 and 2, and works via the same mechanism as olaparib described above and illustrated in Figure 1. Data suggests that PARP inhibitors are especially effective in patients with mutations in DNA repair mechanisms, such as those carrying BRCA mutations [24]. The ARIEL2 trial is investigating specific biomarkers like these to evaluate which, if any, subgroups of the 180 ovarian cancer patients may be sensitive to rucaparib treatment [25]. This data will be incorporated into ARIEL3, a current, randomized, double-blind study phase III trial comparing rucaparib treatment to placebo in 540 ovarian cancer patients [26]. 


\section{Niraparib (MK-4827)}

Niraparib, like the other PARP inhibitors above in Figure 1, blocks poly(ADP-ribose) polymerases 1 and 2 from repairing single-strand breaks in damaged DNA. While most PARP inhibitors exhibit similar catalytic inhibitory properties, it is important to note the differences in potency among drugs in this class [27]. Many propose this discrepancy is due to each drug's individual ability to trap the PARPs at the damaged DNA site. Interestingly, the trapped PARP-DNA complex that forms is more cytotoxic than the single-strand DNA break itself, associating the potency of PARP inhibitors to their ability to form these complexes $[27,28]$. Murai, et al. demonstrated that niraparib was the most potent of the PARP inhibitors due to its superior ability in forming the trapped PARP-DNA complex [28].

A large, 100 patient, phase I trial evaluated niraparib in BRCA $1 / 2$ mutation-carrying (BRCA-MC) patients with high-grade serous ovarian cancer (HGSOC) $(\mathrm{n}=49)$, among others cancers. Of these ovarian cancer patients, $45 \%$ had a partial response from niraparib (60 mg daily). The platinum-sensitive HGSOC subgroup had a response rate almost double that of the platinum-resistant group ( $60 \%$ vs. $33 \%$, respectively), with a median time of response of 429 compared to 340 days. The most common adverse events were grade $1 / 2$ anemia (48\%), fatigue (42\%), nausea (42\%), and thrombocytopenia (35\%). The maximum tolerated dose was determined to be $300 \mathrm{mg}$ daily [29]. Niraparib is currently being studied as maintenance therapy in the randomized, phase III NOVA trial evaluating daily niraparib $(300 \mathrm{mg})$ in 360 patients with high grade serous, platinum sensitive, relapsed ovarian cancer [30].

\section{MAPK kinase (MEK-1 and -2) inhibitors Selumetinib (AZD6244)}

Selumetinib is a mitogen-activated protein kinase inhibitor that shows preclinical benefit in targeting the MEK oncogenic pathway. The small molecular agent is a protein regulator in activated oncogenic pathways expressed in ovarian cancer patients. Results from a phase II study indicate positive activity in the treatment of ovarian cancer. Fifty-two women received two doses of selumetinib (100 mg daily) in the clinical trial, and grade 4 adverse events were only observed in 3 patients (6\%). Thirty-four $(63 \%)$ of the women in the study had a PFS of more than 6 months, with a median OS of 11 months [31]. Larger phase III studies are being planned to further investigate the use of selumetinib as a viable treatment option in ovarian cancer.

\section{Binimetinib (MEK162)}

Binimetinib is an oral inhibitor of MEK-1 and MEK-2, both of which play an important role in cancer cell proliferation and survival via the RAS/RAF/MEK/ERK signal cascade. Inhibiting this pathway is believed to interrupt growth-factor mediated cell signaling as well as inhibit the production of inflammatory cytokines [32]. Binimetinib is currently the subject of nearly twenty clinical trials, including three phase III trials in ovarian cancer and melanoma [33]. The MILO study is an international, randomized phase III study seeking to compare binimetinib to standard chemotherapy in 300 lowgrade serous ovarian cancer (LGSOC) patients [34]. With LGSOC representing about $10 \%$ of all ovarian cancer diagnoses, chemotherapy response rates remain much lower in this group than in their high-grade counterparts. Even more so, less than $4 \%$ of recurrent LGSOC patients respond to additional chemotherapy [33]. The results of the MILO study are highly anticipated, as women with pretreated, recurrent LGSOC do not currently have a successful treatment option [35].

\section{Topoisomerase I inhibitors \\ Etirinotecan pegol (NKTR-102)}

Etirinotecan pegol is a next-generation topoisomerase I inhibitors. Topoisomerase I inhibitors are typically semisynthetic derivatives of the plant extract camptothecin that prevent DNA from unwinding and therefore impede tumor cells from replicating [36]. When normal topoisomerase I inhibitors like irinotecan and belotecan are quickly dispersed within the body, they not only damage healthy tissues, but also have poor half-lives, and do not sufficiently expose the tumor to the concentrated therapeutic agent. Etirinotecan pegol, instead connects small cytotoxic agents to a macromolecular polymer, using specialized linkers. These linkers are then slowly metabolized, resulting in a continuous, controlled release of the chemotherapy, which works as previously described by inhibiting topoisomerase I, and thus, hindering the division of the tumor cells. Preclinical studies have shown a 300-fold increase in the chemotherapy concentration, within the tumor, when compared to other topoisomerase I inhibitors. Along with this, increased effectiveness in tumor concentrations, the half-life of this agent has improved to 50 days, with activity in circulation throughout the entire cell cycle [37].

Clinical trials are under investigation for the use of this agent in various cancers, including ovarian cancer. One phase II, open-label study evaluated etirinotecan pegol $\left(145 \mathrm{mg} / \mathrm{m}^{2}\right)$, every 14 or 21 days in 71 women with platinum-resistant or refractory ovarian cancer. Of patients receiving the drug every 14 days, $27 \%$ had a RECIST response compared to $22 \%$ for those receiving it every 21 days. CA- 125 responses were $61 \%$ and $52 \%$, respectively, with a median time of 31 days to $50 \%$ decline in CA- 125 . The most common grade 3/4 toxicities included diarrhea ( $22 \%$ vs. $11 \%$, respectively), dehydration ( $14 \%$ vs. $6 \%$, 
respectively), and hypokalemia ( $14 \%$ vs. $6 \%$, respectively) [38]. Further investigation was encouraged by the activity observed in these heavily pretreated patients.

A different randomized, multicenter, phase II trial evaluated etirinotecan pegol $\left(145 \mathrm{mg} / \mathrm{m}^{2}\right)$ every 14 or 21 days in 71 women with platinum-resistant or refractory ovarian cancer. Patients who received the agent every 14 days had a slightly higher response rate and response duration (20\% and 4.1 months, respectively) than those who received it every 21 days $(19 \%$ and 4.0 months, respectively). Median PFS and overall response rates were higher in patients receiving the drug every 21 days (5.3 and 11.7 months, respectively) than those receiving it every 14 days (4.1 and 10.0 months, respectively). The drug was well tolerated with grade $3 /$ 4 toxicities including dehydration (24\%) and diarrhea (23\%). Planning for a phase III investigation of etirinotecan pegol $\left(145 \mathrm{mg} / \mathrm{m}^{2}\right)$ every 21 days is currently underway [39]. Phase III investigation in ovarian cancer has also been encouraged by the recent, positive interim efficacy analysis in the phase III BEACON trial for metastatic breast cancer [40].

\section{Cytotoxic agents \\ Mitotic Inhibitor \\ Paclitaxel poliglumex (CT2103)}

Paclitaxel poliglumex is an agent that utilizes polyglutamate drug delivery technology, similar to that described in etirinotecan pegol above. These polyglutamate molecules are much larger than standard paclitaxel molecules, allowing them to lodge themselves in tumor tissue through leaky tumor vasculature. The drug remains inactive in the bloodstream and is too large to fit through normal vasculature, so it specifically targets only tumor cells. Once inside the tumor tissue, the agent is slowly metabolized by the tumor cells, resulting in the controlled release of the cytotoxic agent. This process reduces toxicity to healthy tissues while simultaneously increasing efficacy [41]. Paclitaxel poliglumex falls into the mitotic inhibitor drug class and is under investigation in the treatment of ovarian cancer [42].

Paclitaxel poliglumex $\left(\mathrm{OPAXIO}^{\mathrm{TM}}\right)$ has completed enrollment in a 1,100 patient, randomized phase III trial comparing 12 cycles of maintenance therapy paclitaxel poliglumex or paclitaxel versus no treatment [43]. The previous phase II data that encouraged this phase III trial came nearly 10 years ago in the study of carboplatin with paclitaxel poliglumex in 82 ovarian cancer patients. The study reported $98 \%$ of patients having a major tumor response with reduction in CA-125 levels, complete responses occurring in $85 \%$ of patients, and partial response in $12 \%$. The most common adverse events were grade $3 / 4$ neutropenia (92\%), thrombocytopenia (55\%), and neuropathy (23\%) [44].

\section{DNA minor groove binders Lurbinectedin (PM1183)}

Lurbinectedin is a synthetic analogue of trabectedin (previously discussed) [1]. Positive phase III results were reported for the combination of trabectedin with pegylated liposomal doxorubicin, increasing PFS and overall response rate in women with relapsed ovarian cancer [45]. Trabectedin became the first marine-derived cancer drug, derived from the colonial tunicate, Ecteinascidia turbinate, and marketed in Europe and Japan as Yondelis ${ }^{\oplus}$ [46]. Lurbinectedin has the same structure as trabectedin, differing only in the $\mathrm{C}$ subunit. Soares et al. found that the modified $C$ subunit did not significantly alter lurbinectedin activity or cytotoxicity, and suggested the new analogue may be useful for altering dosages to increase antitumor activity [47]. Lurbinectedin works by covalently binding the minor groove in DNA. This binding causes the DNA strand to bend, increasing the incidence of double-strand breaks while also interfering with cell cycle processes and the nucleotide excision repair pathway [48].

Early in vivo mouse models demonstrated that singleagent lurbinectedin was effective in treating cisplatinsensitive and cisplatin-resistant ovarian tumor models. Preclinical data also suggested that the combination of lurbinectedin with cisplatin-combined therapy was especially effective in the cisplatin-resistant tumors [49]. A randomized, phase II study of 81 platinum-resistant/refractory ovarian cancer patients compared lurbinectedin to topotecan and found that lurbinectedin had significantly improved OS (10.6 vs 5.7 months), PFS (3.9 vs 2.0 months), and the overall response rate (22\%). In the lurbinectedin treatment arm, $85 \%$ of patients experienced grade $3 / 4$ neutropenia, which was found to be preventable by using a G-CSF blood stimulating factor [50]. With this encouraging data and the success of trabectedin, lurbinectedin has received Orphan Drug status from the FDA and phase III trials in platinum-resistant patients have been planned $[48,50]$.

\section{Therapeutic vaccines \\ EpCAM, CD3, and Fc receptor antibody}

Catumaxomab

Catumaxomab (Removab ${ }^{\oplus}$ ) is classified as a trifunctional antibody, with a structure comprised of an anti-EpCAM antibody and an anti-CD3 antibody. This allows catumaxomab to bind to the antigen EpCAM on tumor cells, the CD3 molecules on T cells, and to the Fc receptor on accessory cells, and in doing so, trigger an antitumor immune response [51]. Catumaxomab was approved in Europe in 2009 for the intraperitoneal treatment of malignant ascites in EpCAM-positive cancer patients, and it is currently in clinical trials in the U.S. [52]. Approximately $10 \%$ of ascites, which is the 
accumulation of fluid in the peritoneal cavity, is caused by cancer and called malignant ascites [53].

An open-label, phase II study of catumaxomab in patients with malignant ascites enrolled 32 women and found almost one-fourth (22.6\%) of patients had at least a $400 \%$ increase in their platinum-free interval after catumaxomab treatment. Patients received catumaxo$\operatorname{mab}(10,20,50,150 \mu \mathrm{g})$ on days $0,3,7$, and 10 . The median OS was 3.6 months, with toxicities that were tolerable and consistent with what would be expected for this type of antibody [54]. Another single-arm phase II study administered one intraoperative $(10 \mu \mathrm{g})$ and four postoperative $(10,20,50,150 \mu \mathrm{g})$ doses of catumaxomab on days $7,10,13$, and 16 . The study found a 3 -year survival benefit in patients who received catumaxomab when compared to a match-pair control group (respective survival rates of $85.4 \%$ and $63.4 \%$ ) [55]. This favorable survival data initiated a phase III trial of 258 EpCAM-positive cancer patients with malignant ascites [56].

\section{Conclusions}

As the inclusion of unconventional agents are increasingly incorporated into clinical trials and practice, the hope is that drug discovery will be encouraged in all areas of cancer therapy, from improving our ability to predict response to chemotherapy, to enhancing the delivery of drugs to targeted tissues. As stated previously in an earlier review [1], with the future of cancer treatment moving towards a more personalized approach, the goal is that an individual profile will be determined, and thus agents used that target key pathways in this individual's cancer.

\section{Competing interests}

Dr. Tewari reports that he does have contracted research with Biogen Idec Amgen, Genentech, US Biotest, and Precision Therapeutics. Dr. Monk discloses that his institution has received research grants from Novartis, Amgen, Genentech, Lilly, Janssen/Johson \& Johnson, Array, and TESARO. Additionally, Dr. Monk reports that he has received honoraria from Roche/Genentech as well are consulting fees from Roche/Genentech, GlaxoSmithKline, Merck, TESARO, Boehringer Ingelheim, and AstraZeneca.

\section{Authors' contributions}

DMC, BJM, and KST all provided writing assistance and general support to SJG in the preparation of the tables, figures, and drafting of the manuscript. All authors read and approved the final manuscript.

\section{Acknowledgements}

The authors would like to thank Daniele A. Sumner, BA for her assistance in editing the manuscript. The authors are solely responsible for the preparation and content of the manuscript.

\section{Author details}

${ }^{1}$ The Division of Gynecologic Oncology, University of Arizona Cancer Center, 500 West Thomas Road, Suite 600, Phoenix, AZ 85013, USA. ${ }^{2}$ Creighton University School of Medicine, St. Joseph's Hospital and Medical Center, A Dignity Health Member, 500 West Thomas Road, Suite 600, Phoenix, AZ 85013, USA. ${ }^{3}$ The Division of Gynecologic Oncology, Department of Obstetrics \& Gynecology, The Chao Family Comprehensive Cancer Center, University of California, Irvine Medical Center, 101 The City Drive South, Building 56, Room 275, Orange, CA 92868, USA.
Received: 20 March 2014 Accepted: 4 August 2014

Published: 30 September 2014

\section{References}

1. Chase DM, Mathur N, Tewari KS: Drug discovery in ovarian cancer. Recent Pat Anticancer Drug Discov 2010, 5:251-260.

2. Du Bois A, Floquet A, Kim JW, Rau J, Del Campo JM, Friedlander M, Pignata S, Fujiwara K, Vergote I, Colombo N, Mirza MR, Monk BJ, Wimberger P, Ray-Coquard I, Zang R, Diaz-Padilla I, Baumann KH, Kim JH, Harter P: Randomized, double-blind, phase III trial of pazopanib versus placebo in women who have not progressed after first-line chemotherapy for advanced epithelial ovarian, fallopian tube, or primary peritoneal cancer (AEOC): Results of an international Intergroup trial (AGO-OVAR16). J Clin Oncol 2013, 31:LBA5503.

3. Krasner CN, Poveda A, Herzog TJ, Vermorken JB, Kaye SB, Nieto A, Claret PL, Park YC, Parekh T, Monk BJ: Patient-reported outcomes in relapsed ovarian cancer: results from a randomized Phase III study of trabectedin with pegylated liposomal doxorubicin (PLD) versus PLD alone. Gynecol Oncol 2012, 127:161-167.

4. Li JL, Harris AL: Crosstalk of VEGF and Notch pathways in tumour angiogenesis: therapeutic implications. Front Biosci 2009, 14:3094-3110.

5. Hoff PM, Hochhaus A, Pestalozzi BC, Tebbutt NC, Li J, Kim TW, Koynov KD, Kurteva G, Pintér T, Cheng Y, van Eyll B, Pike L, Fielding A, Robertson JD, Saunders MP: Cediranib Plus FOLFOX/CAPOX Versus Placebo Plus FOLFOX/CAPOX in Patients With Previously Untreated Metastatic Colorectal Cancer: A Randomized, Double-Blind, Phase III Study (HORIZON II). JCO 2012, 30:3596-3603.

6. Schmoll HJ, Cunningham D, Sobrero A, Karapetis CS, Rougier P, Koski SL, Kocakova I, Bondarenko I, Bodoky G, Mainwaring P, Salazar R, Barker P, Mookerjee B, Robertson J, Van Cutsem E: Cediranib With mFOLFOX6 Versus Bevacizumab With mFOLFOX6 As First-Line Treatment for Patients With Advanced Colorectal Cancer: A Double-Blind, Randomized Phase III Study (HORIZON III). JCO 2012, 30:3588-3595.

7. $\mathrm{NCl}$ Drug Dictionary: cediranib maleate. National Cancer Institute at the National Institutes of Health.

8. AstraZeneca Press Release: AstraZeneca welcomes positive data on the combination of olaparib and cediranib for the treatment of ovarian cancer patients; 2014.

9. Liu J, Barry WT, Birrer MJ, Lee JM, Buckanovich RJ, Fleming GF, Rimel BJ, Buss MK, Nattam SR, Hurteau J, Luo W, Quy P, Obermayer E, Whalen C, Lee $\mathrm{H}$, Winer EP, Kohn EC, Ivy SP, Matulonis U: A randomized phase 2 trial comparing efficacy of the combination of the PARP inhibitor olaparib and the antiangiogenic cediranib against olaparib alone in recurrent platinum-sensitive ovarian cancer. J Clin Oncol 2014, 32:5s.

10. ICON6: Cediranib in Ovarian Cancer. http://www.icon6.org.

11. Hilberg F, Roth GJ, Krssak M, Kautschitsch S, Sommergruber W, TontschGrunt U, Garin-Chesa P, Bader G, Zoephel A, Quant J, Heckel A, Rettig WJ: BIBF 1120: triple angiokinase inhibitor with sustained receptor blockade and good antitumor efficacy. Cancer Res 2008, 68:4774-4782.

12. Nintedanib (BIBF 1120): Boehringer Ingelheim; 2012.

13. Ledermann JA, Hackshaw A, Kaye S, Jayson G, Gabra H, McNeish I, Earl H, Perren T, Gore M, Persic M, Adams M, James L, Temple G, Merger M, Rustin G: Randomized phase II placebo-controlled trial of maintenance therapy using the oral triple angiokinase inhibitor BIBF 1120 after chemotherapy for relapsed ovarian cancer. J Clin Oncol 2011, 29:3798-3804.

14. LUME-Ovar 1: Nintedanib (BIBF 1120) or Placebo in Combination With Paclitaxel and Carboplatin in First Line Treatment of Ovarian Cancer. http:// clinicaltrials.gov/show/NCT01015118.

15. Beaton G, Moree WJ, Rueter JK, Dahl RS, McElligott DL, Goldman P, DeMaggio AJ, Christenson E, Herendeen D, Fowler KW, Huang D, Bertino JE, Bourdon LH, Fairfax DJ, Jiang Q, Reisch HA, Song RH, Zhichkin PE: PARP inhibitors. 2005. US6924284.

16. De Lartigue J: New Life for PARP Inhibitors: Emerging Agents Leave Mark at ASCO. OncLive; 2013. http://www.onclive.com/publications/Oncology-live/2013/August2013/New-Life-for-PARP-Inhibitors-Emerging-Agents-Leave-Mark-at-ASCO.

17. Fong PC, Boss DS, Yap TA, Tutt A, Wu P, Mergui-Roelvink M, Mortimer P, Swaisland H, Lau A, O'Connor MJ, Ashworth A, Carmichael J, Kaye SB, Schellens JH, de Bono JS: Inhibition of poly(ADP-ribose) polymerase in tumors from BRCA mutation carriers. N Engl J Med 2009, 361:123-134. 
18. Ledermann J, Harter P, Gourley C, Friedlander M, Vergote I, Rustin G, Scott C, Meier W, Shapira-Frommer R, Safra T, Matei D, Macpherson E, Watkins C, Carmichael J, Matulonis U: Olaparib maintenance therapy in platinumsensitive relapsed ovarian cancer. N Engl J Med 2012, 366:1382-1392

19. Goodman A: Olaparib Shows Robust Progression-Free Survival Benefit in Patients With BRCA Mutations. The ASCO Post; 2013. http://www.ascopost.com/issues/ september-1,-2013/olaparib-shows-robust-progression-free-survival-benefit-inpatients-with-brca-mutations.aspx; http://ovariancancertrials.com/ovariancancer-studies/solo-2/.

20. Studies of olaparib in ovarian cancer (SOLO). AstraZeneca; 2014 http://ovariancancertrials.com/.

21. Olaparib Monotherapy in Patients With BRCA Mutated Ovarian Cancer Following First Line Platinum Based Chemotherapy. http://clinicaltrials.gov/ show/NCT01844986.

22. Olaparib Treatment in BRCA Mutated Ovarian Cancer Patients After Complete or Partial Response to Platinum Chemotherapy. http://clinicaltrials.gov/show/ NCT01874353.

23. Oza AM, Cibula D, Oaknin A, Poole CJ, Mathijssen RHJ, Sonke GS, Colombo N, Spacek J, Vuylsteke P, Hirte HW, Mahner S, Plante M, Schmalfeldt B, Mackay H, Rowbottom J, Tchakov I, Friedlander M: Olaparib plus paclitaxel plus carboplatin $(\mathrm{P} / \mathrm{C})$ followed by olaparib maintenance treatment in patients (pts) with platinum-sensitive recurrent serous ovarian cancer (PSR SOC): A randomized, open-label phase II study. J Clin Oncol 2012, 30:5001.

24. ARIEL2/ARIEL3: New Clinical Trials for Ovarian Cancer. http://www.arielstudy.com.

25. A Study of Rucaparib in Patients With Platinum-Sensitive, Relapsed, High-Grade Epithelial Ovarian, Fallopian Tube, or Primary Peritoneal Cancer (ARIEL2). http:// clinicaltrials.gov/show/NCT01891344.

26. A Study of Rucaparib as Switch Maintenance Following Platinum-Based Chemotherapy in Patients With Platinum-Sensitive, High-Grade Serous or Endometrioid Epithelial Ovarian, Primary Peritoneal or Fallopian Tube Cancer (ARIEL3). http://clinicaltrials.gov/show/NCT01968213

27. BioMarin Press Release: Five Data Presentations on BioMarin's BMN 673 PARP Inhibitor at the 2013 AACR-NCI-EORTC International Conference on Molecular Targets and Cancer Therapeutics; 2013.

28. Murai J, Huang SY, Das BB, Renaud A, Zhang Y, Doroshow JH, Ji J, Takeda S, Pommier Y: Trapping of PARP1 and PARP2 by Clinical PARP Inhibitors. Cancer Res 2012, 72:5588-5599.

29. Michie CO, Sandhu SK, Schelman WR, Molife LR, Wilding G, Omlin AG, Kansra V, Brooks DG, Martell RE, Kaye SB, De Bono JS, Wenham RM: Final results of the phase I trial of niraparib (MK4827), a poly(ADP)ribose polymerase (PARP) inhibitor incorporating proof of concept biomarker studies and expansion cohorts involving BRCA1/2 mutation carriers, sporadic ovarian, and castration resistant prostate cancer (CRPC). J Clin Oncol 2013, 31:abstr 2513.

30. A Maintenance Study With Niraparib Versus Placebo in Patients With Platinum Sensitive Ovarian Cancer. http://clinicaltrials.gov/show/NCT01847274.

31. Array BioPharma Press Release: Encouraging Selumetinib Results Announced for Phase 2 Trial in Ovarian Cancer; 2012.

32. NCI Drug Dictionary: binimetinib. National Cancer Institute at the National Institutes of Health.

33. Array BioPharma 2013 Annual Report: Low Grade Serous Ovarian Cancer (MEK162 - MEK Inhibitor); 2013.

34. Monk BJ, Grisham RN, Marth C, Banerjee SN, Hilpert F, Coleman RL, PujadeLauraine E, Pignata S, Mirza MR, Oza AM, Del Campo JM, Oehler MK, James A, Christy-Bittel J, Barrett E, Boyd A, Vergote I: The MEK Inhibitor in Low-Grade Serous Ovarian Cancer (MILO)/ENGOT-ov11 study: A multinational, randomized, open-label phase 3 study of binimetinib (MEK162) versus physician's choice chemotherapy in patients with recurrent or persistent low-grade serous carcinomas of the ovary, fallopian tube, or primary peritoneum. J Clin Oncol 2014, 32:5s.

35. Array BioPharma 2013 Annual Report: Our Product Pipeline; 2013.

36. Ewesuedoa RB, Ratain MJ: Topoisomerase I Inhibitors. Oncologist 1997, 2:359-364.

37. NEKTAR: R\&D Pipeline: Etirinotecan pegol (NKTR-102); 2014

38. Vergote IB, Micha JP, Pippitt CH Jr, Rao GG, Spitz DL, Reed N, Dark GG, Garcia A, Maslyar DJ, Rustin GJ: Phase II study of NKTR-102 in women with platinum-resistant/refractory ovarian cancer. J Clin Oncol 2010, 28:5013.

39. Vergote IB, Garcia A, Micha JP, Pippitt CH, Bendell J, Spitz D, Reed N, Dark G, Fracasso PM, Ibrahim EN, Armenio VA, Duska L, Poole C, Gennigens C, Dirix $L Y$, Leung AC, Zhao C, Soufi-Mahjoubi R, Rustin G: Randomized Multicenter Phase II Trial Comparing Two Schedules of Etirinotecan Pegol (NKTR-
102) in Women With Recurrent Platinum-Resistant/Refractory Epithelial Ovarian Cancer. J Clin Oncol 2013, 45:1278.

40. Nektar Therapeutics (NKTR) News: Etirinotecan Pegol (NKTR-102) Passes Interim Efficacy Analysis for BEACON Pivotal Phase 3 Clinical Study in Patients with Metastatic Breast Cancer; 2014.

41. CTI BioPharma: Development: About paclitaxel poliglumex; 2014.

42. NCI Drug Dictionary: paclitaxel polyglutamate. National Cancer Institute at the National Institutes of Health.

43. Paclitaxel or Polyglutamate Paclitaxel or Observation in Treating Patients With Stage III or Stage IV Ovarian Epithelial or Peritoneal Cancer or Fallopian Tube Cancer. http://clinicaltrials.gov/show/NCT00108745.

44. PRNewswire: XYOTAX in Combination with Carboplatin Produces Major Tumor Response in 98 Percent of First-line Ovarian Cancer Patients; 2005.

45. Amgen Press Release: Amgen Announces Top-Line Results Of Phase 3 Trebananib (AMG 386) TRINOVA-1 Trial In Recurrent Ovarian Cancer; 2013.

46. Kroll D: Drug From Sea Creature Proves Promising Against Ovarian Cancer. Forbes; 2014. http://www.forbes.com/sites/davidkroll/2014/06/10/under-thesea-pharmamars-lurbinectedin-beats-topotecan-in-platinum-resistantovarian-cancer/.

47. Soares DG, Machado MS, Rocca CJ, Poindessous V, Ouaret D, Sarasin A, Galmarini CM, Henriques JA, Escargueil AE, Larsen AK: Trabectedin and Its C Subunit Modified Analogue PM01183 Attenuate Nucleotide Excision Repair and Show Activity toward Platinum-Resistant Cells. Mol Cancer Ther 2011, 10:1481.

48. PharmaMar (Zeltia Group) Press Release: ZELTIA NEWS: Aplidin ${ }^{\circledR}$, Yondelis ${ }^{\circledR}$, PM01183 and PM060184 highlighted at the 2014 Annual Meeting of the American Association for Cancer Research (AACR); 2014.

49. Vidal A, Muñoz C, Guillén MJ, Moretó J, Puertas S, Martínez-Iniesta M, Figueras A, Padullés L, García-Rodriguez FJ, Berdiel-Acer M, Pujana MA, Salazar R, Gil-Martin M, Martí L, Ponce J, Molleví DG, Capella G, Condom E, Viñals F, Huertas D, Cuevas C, Esteller M, Avilés P, Villanueva A: Lurbinectedin (PM01183), a new DNA minor groove binder, inhibits growth of orthotopic primary graft of cisplatin-resistant epithelial ovarian cancer. Clin Cancer Res 2012, 18:5399-5411.

50. Poveda A, Berton-Rigaud D, Ray-Coquard IL, Alexandre J, Provansal M, Soto A, Kahatt CM, Szyldergemajn SA, Nieto A, Fernandez C, Alia EG, Casado A, Gonzalez-Martin A, Del Campo JM: Lurbinectedin (PM01183), an active compound in platinum-resistant/refractory ovarian cancer (PRROC) patients: Results of a two-stage, controlled phase II study. J Clin Oncol 2014, 32:5s.

51. Chelius D, Ruf P, Gruber P, Plöscher M, Liedtke R, Gansberger E, Hess J, Wasiliu M, Lindhofer $\mathrm{H}$ : Structural and functional characterization of the trifunctional antibody catumaxomab. MAbs 2010, 2:309-319.

52. Fresenius SE: Life Sciences Online; 2009. TRION Pharma: Trifunctional Antibody Catumaxomab Kills Cancer Stem Cells.

53. Cancer.net: Fluid in the Abdomen or Ascites; 2014

54. Berek JS, Edwards RP, Parker L, DeMars LR, Herzog TJ, Lentz SS, Morris R, Akerley WL, Holloway RW, Method M, Plaxe SC, Walker JL, Schindler T, Schulze E, Krasner CN: Catumaxomab treatment of malignant ascites in patients with chemotherapy-refractory ovarian cancer: A phase II study. J Clin Oncol 2011, 29:5048.

55. Pietzner $K$, Chekerov R, Reinthaller A, Reimer D, Reimer T, AngleitnerBoubenizek L, Tschirschmann M, Lindhofer H, Braicu El, Fotopoulou C, Sehouli J: A matched pair analysis of intra- and postoperative catumaxomab in patients with ovarian cancer from a multicenter, singlearm phase II trial versus a consecutive single-center collective of ovarian cancer patients without immunotherapy. J Clin Oncol 2012, 30:5080.

56. Study in EpCAM Positive Patients With Symptomatic Malignant Ascites Using Removab Versus an Untreated Control Group. http://clinicaltrials.gov/ct2/ show/NCT00836654.

doi:10.1186/2053-6844-1-3

Cite this article as: Gibson et al:: Updates on drug discovery in ovarian cancer. Gynecologic Oncology Research and Practice 2014 1:3. 